OPEN ACCESS

Edited by:

Tang Zhonglin,

Agricultural Genomics Institute

at Shenzhen, Chinese Academy

of Agricultural Sciences, China

Reviewed by:

Andrew Craig,

Queen's University, Canada

Zhigang Tu,

Jiangsu University, China

*Correspondence: Satoshi Inoue sinoue@tmig.or.jp

Specialty section:

This article was submitted to Cell Growth and Division,

a section of the journal

Frontiers in Cell and Developmental

Biology

Received: 15 December 2020 Accepted: 06 April 2021

Published: 29 April 2021

Citation:

Takeiwa T, Ikeda K, Horie-Inoue $K$ and Inoue S (2021) Mechanisms

of Apoptosis-Related Long

Non-coding RNAs in Ovarian Cancer.

Front. Cell Dev. Biol. 9:641963.

doi: $10.3389 /$ fcell.2021.641963

\section{Mechanisms of Apoptosis-Related Long Non-coding RNAs in Ovarian Cancer}

\author{
Toshihiko Takeiwa', Kazuhiro Ikeda' ${ }^{1}$, Kuniko Horie-Inoue ${ }^{1}$ and Satoshi Inoue 1,2* \\ 'Division of Systems Medicine \& Gene Therapy, Saitama Medical University, Saitama, Japan, ${ }^{2}$ Department of Systems \\ Aging Science and Medicine, Tokyo Metropolitan Institute of Gerontology, Tokyo, Japan
}

Ovarian cancer is a health-threatening malignancy of ovary in female reproductive systems and one of the most common gynecological malignancies worldwide. Due to rare early symptoms, ovarian cancers are often diagnosed at advanced stages and exhibit poor prognosis. Thus, efforts have been paid to develop alternative diagnostic and therapeutic strategies for the disease. Recent studies have presented that some long non-coding RNAs (IncRNAs) play roles in apoptosis of ovarian cancer cells through various mechanisms involved in the regulation of transcription factors, histone modification complexes, miRNAs, and protein stability. Because evasion of apoptosis in cancer cells facilitates to promote tumor progression and therapy resistance, apoptosis regulatory mechanisms of IncRNAs may be promising new targets in ovarian cancer. In this review, we introduce the recent findings in regard to the molecular mechanisms of apoptosis-related IncRNAs in ovarian cancer cells.

Keywords: ovarian cancer, ovary, apoptosis, long non-coding RNA, transcriptional regulation, histone modification complex, competing endogenous RNA

\section{OVARY AND OVARIAN CANCER}

The ovary is a female reproductive organ where oocyte development occurs (Motta et al., 1997; Virant-Klun, 2015; Yadav et al., 2018) and functions as an endocrine organ involved in the synthesis of the female sex steroid hormones and the regulation of reproduction such as the menstrual cycle, pregnancy, and lactation (Hiller-Sturmhöfel and Bartke, 1998). Thus, the health of ovaries is essential for reproduction and women's lives, rendering finding cures to ovarian diseases crucial. Ovarian cancer is one of the most common gynecological cancers (Momenimovahed et al., 2019). The GLOBOCAN 2018 data estimates 300,000 new cases of ovarian cancer and over 180,000 ovarian cancer-related deaths per year worldwide (Bray et al., 2018). Ovarian cancer is a heterogeneous disease and classified by type of originated cell. Epithelial ovarian cancer (EOC) is the most common ovarian cancer ( 90\%) (Rojas et al., 2016; Momenimovahed et al., 2019). The disease is often advanced at diagnosis due to lack of early symptoms and the 5-year causespecific survival rate is $<50 \%$ (Siegel et al., 2018; Torre et al., 2018; Trinidad et al., 2020). Based on the current limitations, alternative diagnostic and therapeutic approaches for ovarian cancer remain to be explored. 


\section{APOPTOSIS IN OVARY AND OVARIAN CANCER}

Apoptosis is a process of programmed cell death triggered by intrinsic or extrinsic signals (Wong, 2011). Intrinsic signals are initiated by cellular stresses. These signals increase the mitochondrial permeability and release of the pro-apoptotic factors such as cytochrome-c, resulting in activation of cysteineaspartic acid proteases (caspases), which are essential enzymes for apoptosis execution. Meanwhile, extrinsic signals are mediated by death receptor signaling pathways. Death receptors, their ligands, and adaptor proteins form the death-inducing signaling complex (DISC), which triggers caspase activation (Wong, 2011).

Apoptosis plays physiological roles in normal ovary functions such as follicular atresia and corpus luteum regression (Vaskivuo and Tapanainen, 2003; Yadav et al., 2018). In malignant tumors, evasion of apoptosis facilitates cancer cell survival and tumor progression (Wong, 2011; Binju et al., 2019), thus efforts have been paid for cancer strategies to discover the molecules to exert apoptosis in cancer cells whereas not in normal cells. For ovarian cancer treatment, small chemicals that modulate apoptosis-related proteins such as inhibitors of apoptotic proteins (IAPs) have entered clinical trials (Binju et al., 2019).

In terms of apoptosis pathways in cancers, several mechanisms of apoptosis-related genes have been well characterized. Transcription factors such as E2F family proteins, nuclear factor kappa $\mathrm{B}(\mathrm{NF}-\mathrm{\kappa} \mathrm{B})$ proteins, and signal transducer and activator of transcription (STAT) family proteins modulate apoptosis via regulating transcription of apoptosis-related genes (Bours et al., 2000; Crosby and Almasan, 2004; Karin, 2006; Kim and Lee, 2007; Kent and Leone, 2019; Verhoeven et al., 2020). Histone modification complexes such as polycomb repressive complex 1/2 (PRC1/2) affect transcription of apoptosis-related genes through histone methylation (Cao et al., 2011; Wang W. et al., 2015; Christofides et al., 2016). Apoptosis-related genes are also modulated by post-transcriptional gene regulation mechanism, such as through miRNAs that regulate apoptosisrelated gene mRNAs (Di Leva et al., 2014; Pistritto et al., 2016; Si et al., 2019). Ubiquitin-mediated protein degradation systems are also involved in apoptosis (Zhang et al., 2004; Hoeller and Dikic, 2009; Yang et al., 2009), as some E3 ubiquitin ligases are involved in ubiquitination of apoptosis-related proteins (Hoeller and Dikic, 2009; Yang et al., 2009, 2018; Woo and Kwon, 2019).

Considering the importance of apoptosis in cancer pathophysiology, strategies targeting these apoptosis regulatory mechanisms may contribute to the development of novel ovarian cancer therapies.

\section{LONG NON-CODING RNA (IncRNA)}

Long non-coding RNAs (lncRNAs) are defined as $>200$ nt transcripts that do not encode proteins and tens of thousands of lncRNA transcripts are identified throughout the human genome, the majority with unknown function. However, functional studies of some lncRNAs have revealed that they have a wide range of functions. For example, lncRNAs regulate transcription and chromatin remodeling by modulating the recruitment of transcription factors and PRC to specific genomic loci. Furthermore, lncRNAs are involved in gene regulation at post-transcriptional levels through interacting with mRNAs, miRNAs, and proteins (Marchese et al., 2017). Intriguingly, IncRNAs play important roles in pathophysiology of various cancers (Takayama and Inoue, 2016; Misawa et al., 2017; Arun et al., 2018; Mitobe et al., 2018; Kamada et al., 2020; Takeiwa et al., 2020). Particularly, several lncRNAs have been suggested to regulate the apoptosis of ovarian cancer cells (Figure 1 and Table 1). In the following sections, we will describe some apoptosis-related lncRNAs in ovarian cancer cells according to their mechanisms.

\section{Apoptosis-Related LncRNAs Regulating Transcription Factors}

In this section, we will introduce an apoptosis-promotive lncRNA growth arrest-specific 5 (GAS5) and apoptosis-suppressive IncRNAs non-coding RNA in the aldehyde dehydrogenase $1 A$ pathway (NRAD1)/long intergenic non-coding RNA 00284 (LINC00284) and a non-coding variant of ceruloplasmin (CP) (lncRNA ceruloplasmin; NRCP).

\section{GAS5}

Growth arrest-specific 5 is downregulated in ovarian cancer, with this low expression associated with shorter disease-free period and lower overall survival rate of ovarian cancer patients (Gao et al., 2015; Li et al., 2016; Zhao et al., 2018; Long et al., 2019). GAS5 overexpression promotes apoptosis of ovarian cancer cells such as A2780, HEY, OVCAR3, and SKOV3, and increases the sensitivity of HEY and SKOV3 cells to the anticancer agent cisplatin (Gao et al., 2015; Li et al., 2016; Zhao et al., 2018; Long et al., 2019). A functional study has shown that GAS5 recruits the E2F4 transcription factor to the poly(ADP-ribose) polymerase 1 (PARP1) promoter, repressing PARP1 transcription in HEY and SKOV3 cells (Long et al., 2019; Figure 1A). GAS5-mediated PARP1 repression might contribute to apoptosis by downregulating the mitogen-activated protein kinase (MAPK) pathway, but direct evidence will be required in the future study.

\section{NRAD1/LINC00284}

Non-coding RNA in the aldehyde dehydrogenase 1 A pathway/long intergenic non-coding RNA 00284 is highly expressed in ovarian cancer. NRAD1 overexpression and knockdown experiments have shown that it suppresses the apoptosis of HO8910 and OVCAR3 cells. Functional analyses using HO8910 cells have suggested that NRAD1 binds to NF-KB subunit 1 (NF$\kappa B 1)$ transcription factor and induces NF- $\mathrm{B} 1$-mediated transcriptional repression of mesoderm specific transcript (MEST), contributing to the suppression of apoptosis (Ruan and Zhao, 2019; Figure 1A). However, since a previous study has reported that HO8910 is a cross-contaminated cell line, this mechanism is needed to be verified using other ovarian cancer models (Ye et al., 2015). 
A

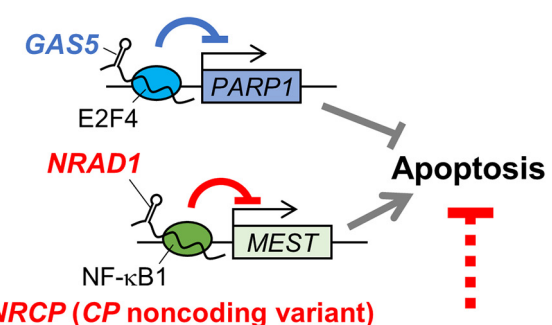

$N R C P$ (CP noncoding variant)

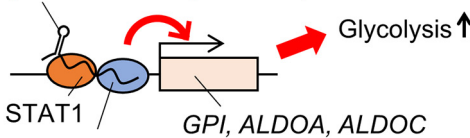

RNA polymerase II

GPI, ALDOA, ALDOC

C

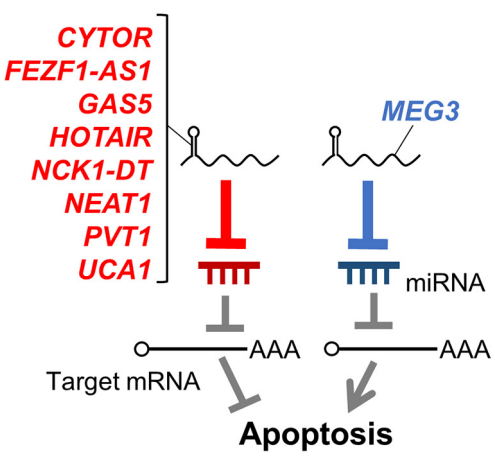

B FALEC

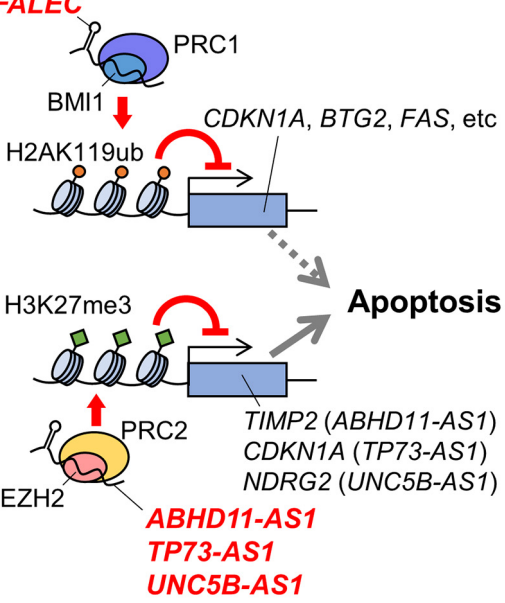

D

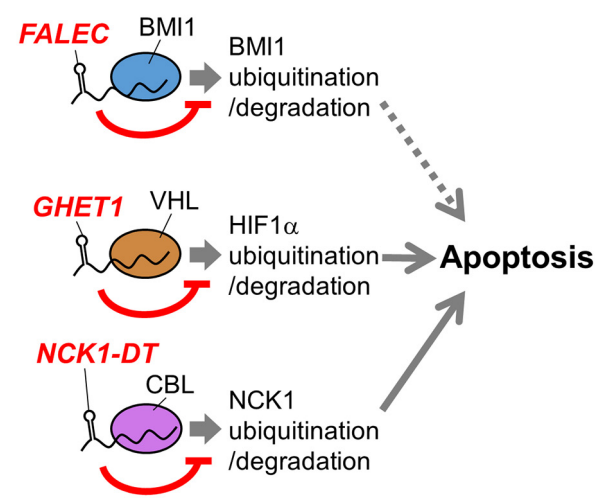

FIGURE 1 | Schematic representation of mechanisms of apoptosis-related IncRNAs in ovarian cancer. LncRNAs involved in apoptosis of ovarian cancer cells via regulating transcription factors (A), histone modification complexes (B), miRNAs (C), and protein stability (D) are shown. Names of IncRNAs promoting and suppressing apoptosis are shown in blue and red, respectively. In addition, apoptosis-promotive or suppressive functions of IncRNAs are shown in blue or red lines, respectively. Potential apoptosis-associated biological pathways are shown in dotted lines. ABHD11-AS1, abhydrolase domain containing 11 antisense RNA 1; ALDOA, aldolase, fructose-bisphosphate A; ALDOC, aldolase, fructose-bisphosphate C; BMl1, B lymphoma Mo-MLV insertion region 1 homolog; BTG2, B-cell translocation gene 2; CBL, casitas B-lineage lymphoma: CDKN1A, cyclin dependent kinase inhibitor 1A; CP, ceruloplasmin; CYTOR, cytoskeleton regulator RNA; EZH2, enhancer of zeste homolog 2; FALEC, focally amplified long non-coding RNA in epithelial cancer; FEZF1-AS1, fasciculation and elongation protein zeta family zinc finger 1 antisense RNA 1; GAS5, growth arrest-specific 5; GHET1, gastric carcinoma high expressed transcript 1; GPI, glucose-6-phosphate isomerase; H2AK119ub, the ubiquitination at the 119th lysine residue of the histone H2A; H3K27me3, the tri-methylation at the 27th lysine residue of the histone H3; HIF1 $\alpha$, hypoxia-inducible factor 1 $\alpha$; HOTAIR, HOX transcript antisense RNA; MEG3, maternally expressed gene 3; MEST, mesoderm specific transcript; NCK1, non-catalytic region of tyrosine kinase adaptor protein 1; NCK1-DT, NCK1 divergent transcript; NDRG2, n-myc downstream-regulated gene 2; NEAT1, nuclear enriched abundant transcript 1; NF-kB1, nuclear factor kappa B subunit 1; NRAD1, non-coding RNA in the aldehyde dehydrogenase 1A pathway; NRCP, IncRNA ceruloplasmin; PARP1, poly(ADP-ribose) polymerase 1; PVT1, plasmacytoma variant translocation 1; STAT1, signal transducer and activator of transcription 1; TIMP2, tissue inhibitor of metalloproteinase 2; TP73-AS1, tumor protein p73 antisense RNA 1; UCA1, urothelial carcinoma associated 1; UNC5B-AS1, uncoordinated 5 netrin receptor $B$ antisense RNA 1.

\section{CP Non-coding Variant (NRCP)}

$N R C P$ is a non-coding splice variant of the ceruloplasmincoding gene that is upregulated in ovarian cancer (Rupaimoole et al., 2015). High NRCP expression levels correlate with shorter overall survival in patients with ovarian cancer, while NRCP knockdown induces apoptosis in A2780 and SKOV3 cells (Rupaimoole et al., 2015). NRCP binds to RNA polymerase II and STAT1 transcription factor, and promotes glycolysis in A2780 and SKOV 3 cells by upregulating glycolysis pathway genes such as glucose-6-phosphate isomerase (GPI), aldolase, fructosebisphosphate A (ALDOA), and aldolase, fructose-bisphosphate $C$ (ALDOC) via STAT1 (Rupaimoole et al., 2015; Figure 1A).
These results suggest a possibility that $N R C P$ may modulate apoptosis by regulating cancer metabolism. NRCP is not annotated in National Center for Biotechnology Information (NCBI) Reference Sequence (RefSeq) database (on Feb 3rd, 2021) and requires further characterization of sequences and expression profiles.

\section{Apoptosis-Related LncRNAs Regulating Histone Modification Complexes}

In this section, we will describe the following apoptosissuppressive IncRNAs: abhydrolase domain containing 11 
TABLE 1 | Mechanisms of apoptosis-related IncRNAs in ovarian cancer cells.

\begin{tabular}{|c|c|c|c|c|}
\hline IncRNA & Chr. location & Roles in ovarian cancer cells/xenograft tumors & $\begin{array}{l}\text { Clinical relevance in ovarian } \\
\text { cancer tissues/patients }\end{array}$ & $\begin{array}{l}\text { Effects on } \\
\text { apoptosis }\end{array}$ \\
\hline GAS5 & 1q25 & $\begin{array}{l}\downarrow \text { in } \mathrm{A} 2780 / \mathrm{HEY} / \mathrm{HO} 8910 \text { a/OVCAR3/SKOV3 cells } \\
\text { Binds to E2F4 and represses PARP1 in HEY/SKOV3 cells } \\
\text { Sponges miR-196a-5p to upregulate HOXA5 in primary tumor } \\
\text { cells from HGSOV tissues and A2780/OVCAR3 cells } \\
\text { Represses PARP1, growth and cisplatin resistance in SKOV3 } \\
\text { tumors }\end{array}$ & $\begin{array}{l}\downarrow \text { in tumor tissues } \\
\downarrow \text { is correlated with shorter } \\
\text { DFS/OS }\end{array}$ & + \\
\hline NRAD1/LINC00284 & $13 q 14$ & $\begin{array}{l}\uparrow \text { in A2780/CAOV3/HO8910/OVCAR3/SKOV3 cells } \\
\text { Binds to NF-kB1 and represses MEST in HO8910 cells } \\
\text { Promotes growth of HO8910 tumors }\end{array}$ & $\uparrow$ in ovarian cancer tissues & - \\
\hline $\begin{array}{l}C P \text { non-coding } \\
\text { variant }(N R C P)\end{array}$ & $3 q 24-25$ & $\begin{array}{l}\uparrow \text { in A2780/IGROV1/OVCAR3/SFMAR/SFWAS/SKOV3 cells } \\
\text { Binds to RNA Pol II and STAT1, upregulates GPI, ALDOA, and } \\
\text { ALDOC } \\
\text { Promotes growth and metastasis of A2780/SKOV3 tumors }\end{array}$ & $\begin{array}{l}\uparrow \text { in tumor tissues } \\
\uparrow \text { is correlated with shorter OS }\end{array}$ & - \\
\hline$A B H D 11-A S 1$ & $7 q 11$ & $\begin{array}{l}\uparrow \text { in HO8910/OVCA429 cells } \\
\text { Upregulates RhoC in A2780/OVCAR3 cells } \\
\text { Binds to EZH2 and represses TIMP2 in HO8910/OVCA429 cells } \\
\text { Promotes growth and metastasis of A2780 tumors }\end{array}$ & $\uparrow$ in tumor tissues & - \\
\hline FALEC/FAL1 & $1 q 21$ & $\begin{array}{l}\text { Promotes PRC1-mediated repression of CDKN1A, BTG2, and } \\
\text { FAS in A2780 cells } \\
\text { Binds to and stabilizes BMl1 in A2780 cells } \\
\text { Promotes A2780 tumor growth }\end{array}$ & $\begin{array}{l}\uparrow \text { and copy number gain } \\
\text { correlated with shorter OS }\end{array}$ & - \\
\hline TP73-AS1 & $1 \mathrm{p} 36$ & $\begin{array}{l}\uparrow \text { in CAOV3/HO8910/OV420/SKOV3 cells } \\
\text { Binds to EZH2 and represses CDKN1A in SKOV3 cells } \\
\text { Promotes SKOV3 tumor growth }\end{array}$ & $\begin{array}{l}\uparrow \text { in tumor tissues } \\
\uparrow \text { is correlated with poor } \\
\text { prognosis }\end{array}$ & - \\
\hline UNC5B-AS1 & $10 q 22$ & $\begin{array}{l}\uparrow \text { in } \mathrm{A} 2780 / \mathrm{ES} 2 / \mathrm{SKOV} 3 \text { cells } \\
\text { Binds to EZH2 and promotes NDRG2 in ES2/SKOV3 cells }\end{array}$ & $\uparrow$ in tumor tissues & - \\
\hline FEZF1-AS1 & $7 q 31$ & $\begin{array}{l}\uparrow \text { in A2780/COC1/PEO1/SKOV3 cells } \\
\text { Sponges miR-130a-5p, upregulates SOX4 in COC1/SKOV3 cells }\end{array}$ & $\begin{array}{l}\uparrow \text { in tumors and serum } \\
\uparrow \text { is correlated with shorter OS }\end{array}$ & - \\
\hline HOTAIR & $12 q 13$ & $\begin{array}{l}\uparrow \text { in cisplatin-resistant A2780/SKOV3 cells } \\
\text { Sponges miR-138-5p, upregulates EZH2 and SIRT1 in } \\
\text { A2780/SKOV3 cells } \\
\text { Promotes A2780 tumor growth }\end{array}$ & $\begin{array}{l}\uparrow \text { in tumor tissues } \\
\uparrow \text { is correlated with shorter OS in } \\
\text { HGSOV patients }\end{array}$ & - \\
\hline CYTOR/LINC00152 & $2 \mathrm{p} 11$ & $\begin{array}{l}\uparrow \text { in A2780/HO8910/SKOV3 cells } \\
\text { Sponges miR-125b to upregulate MCL } 1 \text { in A2780/SKOV3 cells } \\
\text { Increases MCL1 level and SKOV3 tumor growth }\end{array}$ & $\begin{array}{l}\uparrow \text { in tumor tissues } \\
\uparrow \text { is correlated with shorter OS }\end{array}$ & - \\
\hline MEG3 & $14 q 32$ & $\begin{array}{l}\downarrow \text { in OVCAR3/OVCAR5/OVCAR8/SKOV3 cells } \\
\text { Sponges miR-205-5p in OVCAR8/SKOV3 cells }\end{array}$ & $\downarrow$ in tumor tissues & + \\
\hline $\begin{array}{l}\text { NCK1-DT/NCK1- } \\
\text { AS1 }\end{array}$ & $3 q 22$ & $\begin{array}{l}\uparrow \text { in CAOV3/OVCAR3/SKOV3/SNU119/SUN8 cells } \\
\text { Sponges miR-137 to upregulate NCK1 in CAOV3/SKOV3 cells } \\
\text { Prevents CBL-mediated NCK1 degradation in CAOV3/SKOV3 } \\
\text { cells }\end{array}$ & $\uparrow$ in tumor tissues & - \\
\hline NEAT1 & $11 q 13$ & $\begin{array}{l}\uparrow \text { in A2780/CAOV3/ES2/HO8910/OV90/OVCAR3/SKOV3 cells } \\
\uparrow \text { in paclitaxel-resistant HeyA8/SKOV3 cells versus parental cells } \\
\uparrow \text { in cisplatin-resistant A2780/SKOV3 cells versus parental cells } \\
\text { Sponges miR-34a-5p, upregulates BCL2 in OVCAR3/SKOV3 } \\
\text { cells } \\
\text { Sponges miR-4500, upregulates BZW1 in CAOV3/ES2 cells } \\
\text { Sponges miR-194, upregulates ZEB1 in HeyA8/SKOV3 cells } \\
\text { Sponges miR-770-5p, upregulates PARP1 in A2780/SKOV3 cells } \\
\text { Promotes growth of SKOV3/A2780 tumors and paclitaxel } \\
\text { resistance }\end{array}$ & $\begin{array}{l}\uparrow \text { in tumor tissues } \\
\uparrow \text { in cisplatin and } \\
\text { paclitaxel-resistant cancer tissues } \\
\uparrow \text { is correlated with shorter OS }\end{array}$ & - \\
\hline PVT1 & $8 q 24$ & $\begin{array}{l}\uparrow \text { in A2780/OVCAR3/TOV112D cells } \\
\text { Sponges miR-543, upregulates SERPIN/1 in OVCAR3/TOV112D } \\
\text { cells }\end{array}$ & $\begin{array}{l}\uparrow \text { in tumor tissues } \\
\uparrow \text { is correlated with shorter OS }\end{array}$ & - \\
\hline UCA1 & 19p13 & $\begin{array}{l}\uparrow \text { in A2780/HeyA8/OAW42/OVCAR4/SKOV3 cells } \\
\uparrow \text { in paclitaxel-resistant HeyA8/SKOV3 cells } \\
\uparrow \text { in cisplatin-resistant A2780/SKOV3 cells } \\
\text { Sponges miR-129, upregulates ABCB1 in HeyA8/SKOV3 cells } \\
\text { Sponges miR-654-5p, upregulates SIK2 in HeyA8/SKOV3 cells } \\
\text { Sponges miR-143, upregulates FOSL2 in A2780/SKOV3 cells }\end{array}$ & $\begin{array}{l}\uparrow \text { in tumor tissues } \\
\uparrow \text { in tumors and serum exosomes } \\
\text { of patients with cisplatin-resistant } \\
\text { cancers }\end{array}$ & - \\
\hline
\end{tabular}


TABLE 1 | Continued

\begin{tabular}{|c|c|c|c|c|}
\hline IncRNA & $\begin{array}{l}\text { Chr. } \\
\text { location }\end{array}$ & Roles in ovarian cancer cells/xenograft tumors & $\begin{array}{l}\text { Clinical relevance in ovarian } \\
\text { cancer tissues/patients }\end{array}$ & $\begin{array}{l}\text { Effects on } \\
\text { apoptosis }\end{array}$ \\
\hline GHET1 & $7 q 36$ & $\begin{array}{l}\uparrow \text { in } 3 \mathrm{AO} / \mathrm{A} 2780 / \mathrm{OVCAR} 3 / \mathrm{SKOV} 3 \text { cells } \\
\text { Prevents VHL-mediated HIF1 } \alpha \text { degradation in A2780/SKOV3 cells }\end{array}$ & $\begin{array}{l}\uparrow \text { in tumor tissues } \\
\uparrow \text { is correlated with increased } \\
\text { tumor size and distant } \\
\text { metastasis }\end{array}$ & - \\
\hline
\end{tabular}

although HO8910 cell line was used as an ovarian cancer cell model in these studies, a previous study has reported that it is a cross-contaminated cell line. ABCB1, ATP binding cassette subfamily B member 1; ABHD11-AS1, abhydrolase domain containing 11 antisense RNA 1; ALDOA, aldolase, fructose-bisphosphate A; ALDOC, aldolase, fructose-bisphosphate C; BCL2, B-cell lymphoma 2; BMI1, B lymphoma Mo-MLV insertion region 1 homolog; BTG2, B-cell translocation gene 2; BZW1, basic leucine zipper and W2 domain-containing protein 1; CBL, casitas B-lineage lymphoma; CDKN1A, cyclin dependent kinase inhibitor 1A; CP, ceruloplasmin; CYTOR, cytoskeleton regulator RNA; DFS, disease-free survival; EZH2, enhancer of zeste homolog 2; FAL1, focally amplified IncRNA on chromosome 1; FALEC, focally amplified long non-coding RNA in epithelial cancer; FEZF1-AS1, fasciculation and elongation protein zeta family zinc finger 1 antisense RNA 1; FOSL2, Fos-related antigen 2; GAS5, growth arrest-specific 5; GHET1, gastric carcinoma high expressed transcript 1; GPI, glucose-6-phosphate isomerase; HGSOV, high-grade serous ovarian cancer; HIF1 $\alpha$, hypoxia-inducible factor 1 $\alpha$; HOTAIR, HOX transcript antisense RNA; HOXA5, homeobox A5; LINC00152, long intergenic non-coding RNA 00152; LINC00284, long intergenic non-coding RNA 00284; MCL1, myeloid cell leukemia 1; MEG3, maternally expressed gene 3; MEST, mesoderm specific transcript; NCK1, non-catalytic region of tyrosine kinase adaptor protein 1; NCK1-AS1, NCK1 antisense RNA 1; NCK1-DT, NCK1 divergent transcript; NDRG2, n-myc downstream-regulated gene 2; NEAT1, nuclear enriched abundant transcript 1; NRAD1, non-coding RNA in the aldehyde dehydrogenase 1A pathway; NRCP, IncRNA ceruloplasmin; OS, overall survival;

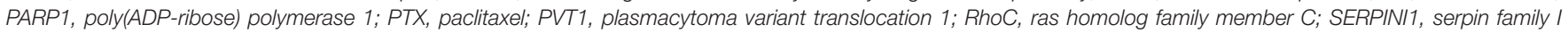

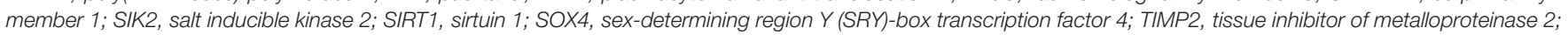

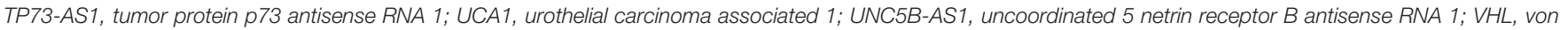
Hippel-Lindau tumor suppressor; ZEB1, zinc finger E-box binding homeobox 1.

antisense RNA 1 (ABHD11-AS1), focally amplified long noncoding RNA in epithelial cancer (FALEC)/focally amplified lncRNA on chromosome 1 (FAL1), tumor protein p73 antisense RNA 1 (TP73-AS1), and uncoordinated 5 netrin receptor $B$ antisense RNA 1 (UNC5B-AS1).

\section{ABHD11-AS1}

Abhydrolase domain containing 11 antisense RNA 1 is upregulated in ovarian cancer (Wu et al., 2017; Zeng et al., 2019). A functional study has shown that ABHD11-AS1 modulates the expression of ras homolog family member $\mathrm{C}$ (RhoC) by an unknown mechanism, suppressing apoptosis in A2780 and OVCAR3 cells (Wu et al., 2017). Another functional study has shown that ABHD11-AS1 binds to enhancer of zeste homolog 2 (EZH2), a component of PRC2. ABHD11-AS1 facilitates tri-methylation at the 27th lysine residue of the histone $\mathrm{H} 3$ protein $(\mathrm{H} 3 \mathrm{~K} 27 \mathrm{me} 3)$ on the tissue inhibitor of metalloproteinase 2 (TIMP2) promoter, as mediated by PRC2, and likewise suppresses TIMP2 expression in HO8910 cells and OVCA429 ovarian cancer cells (Figure 1B). TIMP2 suppression mediated by ABHD11-AS1 promotes the proliferation of OVCA429 cells, suggesting that ABHD11-AS1 may also modulate apoptosis by this mechanism (Zeng et al., 2019).

\section{FALEC/FAL1}

Focally amplified IncRNA in epithelial cancer/focally amplified IncRNA on chromosome 1 was initially identified as an lncRNA whose gene copy number increased in multiple types of cancers, including ovarian cancer (Hu et al., 2014). Its high expression level and gain in genomic copy number correlate with a shorter overall survival rate of late-stage ovarian cancer patients (Hu et al., 2014). A functional study using A2780 cells has suggested that FALEC binds to a component of PRC1, B lymphoma Mo-MLV insertion region 1 homolog (BMI1) protein, and recruits PRC1 to the promoters of genes such as cyclin dependent kinase inhibitor $1 A$ (CDKN1A), Bcell translocation gene 2 (BTG2), and FAS. Subsequently, PRC1 mediates the ubiquitination at the 119th lysine residue of the histone $\mathrm{H} 2 \mathrm{~A}$ (H2AK119ub) on these promoter regions and the suppression of these genes (Figure 1B). The FALEC/PRC1 complex target genes such as CDKN1A, BTG2, and FAS are suggested to be involved in apoptosis regulation (El-Deiry, 2001; Mao et al., 2015). Thus, FALEC can be a regulator of ovarian cancer apoptosis.

\section{TP73-AS1}

Tumor protein p73 antisense RNA 1 is upregulated in EOC and associated with poor prognosis in EOC patients (Li Y. et al., 2019). A recent study has shown that TP73-AS1 knockdown induces apoptosis of SKOV3 cells, suppressing the proliferation in in vitro culture and the xenograft tumor formation in athymic mice. In contrast, TP73-AS1 overexpression suppresses apoptosis in CAOV3 ovarian cancer cells. Functional analyses have suggested that TP73-AS1 epigenetically suppresses CDKN1A expression by recruiting PRC2 to its promoter (Figure 1B) and modulates apoptosis of SKOV3 cells through this mechanism (Li Y. et al., 2019).

\section{UNC5B-AS1}

Uncoordinated 5 netrin receptor $B$ antisense RNA 1 is highly expressed in ovarian cancer, and a recent study has shown that its knockdown activates caspase 3 in ES2 and SKOV3 cells, suggesting the apoptosis-suppressive role of UNC5BAS1 (Wang et al., 2020). Moreover, the same study has suggested that $U N C 5 B-A S 1$ promotes PRC2 to repress the $n-m y c$ downstream-regulated gene 2 (NDRG2) expression epigenetically (Figure 1B), which may suppress ovarian cancer cell apoptosis (Wang et al., 2020). This study is limited in the elucidation of the mechanism by which UNC5B-AS1 regulates PRC2 and its in vivo function, and further functional analyses are required. 


\section{Apoptosis-Related LncRNAs Regulating miRNAs}

In the section, we will introduce an apoptosis-promotive lncRNA GAS5 and the following apoptosis-suppressive lncRNAs: fasciculation and elongation protein zeta family zinc finger 1 antisense RNA 1 (FEZF1-AS1), HOX transcript antisense RNA (HOTAIR), non-catalytic region of tyrosine kinase adaptor protein 1 (NCK1) divergent transcript (NCK1-DT)/NCK1 antisense RNA 1 (NCK1-AS1), nuclear enriched abundant transcript 1 (NEAT1), and urothelial carcinoma associated 1 (UCA1).

\section{FEZF1-AS1}

High levels of FEZF1-AS1 are detected in tumor tissues and the serum of EOC patients, with its high expression associated with shorter overall survival of EOC patients (Sun et al., 2020). Moreover, its knockdown promotes apoptosis in COC1 and SKOV3 ovarian cancer cells, suggesting the apoptosissuppressive role of FEZF1-AS1. In vitro analyses of FEZF1-AS1 have shown that it functions as a competing endogenous RNA (ceRNA) for miR-130a-5p, or sponges miR-130a-5p (Figure 1C). Consequently, FEZF1-AS1 upregulates the expression of a miR-130a-5p target gene, sex-determining region $Y$ (SRY)-box transcription factor 4 (SOX4), that promotes proliferation of $\mathrm{COC1}$ and SKOV3 cells and may contribute to apoptosis suppression (Sun et al., 2020). Further analysis of FEZF1-AS1 function, especially in vivo, will clarify its role and significance in apoptosis of ovarian cancer cells.

\section{GAS5}

A recent functional study has suggested that GAS5 functions as a ceRNA for miR-196a-5p to upregulate homeobox A5 (HOXA5), promoting apoptosis of primary tumor cells from high-grade serous ovarian cancer tissues as well as A2780 and OVCAR3 cells (Zhao et al., 2018; Figure 1C).

\section{HOTAIR}

HOX transcript antisense RNA is upregulated in ovarian cancer, and the elevated expression level correlates with the shorter overall survival of ovarian cancer patients (Qiu et al., 2015; Wang Y. et al., 2015; Zhang et al., 2016; Luo et al., 2017; Yu et al., 2018). HOTAIR knockdown induces apoptosis in ovarian cancer cells such as A2780, HeyC2, and OVCA429, and decreases the cisplatin sensitivity of A2780 and SKOV3 cells (Qiu et al., 2015; Wang Y. et al., 2015; Zhang et al., 2016, 2020; Yu et al., 2018). A recent functional study using A2780 and SKOV3 cells has suggested that HOTAIR acts as a ceRNA for miR-138-5p, leading to cisplatin resistance of these cells (Zhang et al., 2020; Figure 1C). This study has shown that HOTAIR/miR-138-5p axis modulates EZH2 and sirtuin 1 (SIRT1) expression, but its biological significance has not been elucidated.

\section{NCK1-DT/NCK1-AS1}

Non-catalytic region of tyrosine kinase adaptor protein 1 divergent transcript is highly expressed in ovarian cancer. Mechanistically, it acts as a ceRNA for $m i R-137$ to upregulate NCK1, which suppresses apoptosis of CAOV3 and SKOV3 cells and enhances their cisplatin resistance (Chang et al., 2020; Figure 1C).

\section{NEAT1}

Nuclear enriched abundant transcript 1 is upregulated in ovarian cancer and is associated with shorter overall survival of ovarian cancer patients (Chen et al., 2016). NEAT1 acts as a ceRNA for $m i R-34 a-5 p$ to upregulate BCL2 and suppresses apoptosis of OVCAR3 and SKOV3 cells (Ding et al., 2017). In addition, NEAT 1 acts as a ceRNA for $m i R-4500$, to upregulate basic leucine zipper and W2 domain-containing protein 1 (BZW1) that suppresses apoptosis of CAOV3 and ES2 cells (Xu et al., 2020), and miR-194 to upregulate the transcription factor zinc finger E-box binding homeobox 1 (ZEB1), promoting resistance to the anticancer agent paclitaxel (PTX) in HeyA8 and SKOV3 cells (An et al., 2017). Furthermore, NEAT1 sponges miR-770-5p, to upregulate PARP1 and increase cisplatin resistance in A2780 cells in vivo (Zhu et al., 2020; Figure 1C).

\section{UCA1}

The IncRNA UCA1 is upregulated in ovarian cancer and is detected in exosomes derived from the serum of ovarian cancer patients (Li Z. et al., 2019; Li et al., 2020). Functional studies have shown that UCA1 acts as a ceRNA for miR-129 and miR-654-5p to upregulate ATP binding cassette subfamily $B$ member 1 (ABCB1) and SALT INDUCIBLE KINASE 2 (SIK2), respectively, which contribute to the suppression of apoptosis and the enhancement of PTX resistance in HeyA8 and SKOV3 cells (Wang et al., 2018; Li et al., 2020). In addition, UCA1 functions as a ceRNA for miR-143 to increase Fos-related antigen 2 (FOSL2), and enhances cisplatin resistance in A2780 and SKOV3 cells (Li Z. et al., 2019; Figure 1C). However, the importance of the function of UCA1 as a ceRNA in vivo has not been fully analyzed.

Recent studies have found that many other lncRNAs modulate ovarian cancer apoptosis through regulating miRNAs. For example, CYTOR/LINC00152 acts as a ceRNA of $m i R-125 b$ to upregulate an antiapoptotic protein MCL1 in A2780 and SKOV3 cells (Chen et al., 2018). PVT1 suppresses apoptosis in OVACAR3 and TOV112D cells by inhibiting $m i R-543$ and increasing a $m i R-543$ target SERPIN1 (Qu et al., 2020). In contrast, MEG3 promotes apoptosis in OVCAR8 and SKOV3 cells by sponging $m i R$ 205-5p (Tao et al., 2020). The detail of lncRNAs regulating miRNAs is also reviewed in other articles (Braga et al., 2020; Salamini-Montemurri et al., 2020).

\section{Apoptosis-Related LncRNAs Regulating Protein Stability}

In the section, we will introduce the following apoptosissuppressive lncRNAs: FALEC/FAL1, gastric carcinoma high expressed transcript 1 (GHET1), and NCK1-DT/NCK1-AS1.

\section{FALEC/FAL1}

As described above, FALEC binds to BMI1 and modulates PRC1 function in A2780 cells. In addition, FALEC stabilizes BMI1 by suppressing ubiquitin-mediated BMI1 protein degradation ( $\mathrm{Hu}$ et al., 2014; Figure 1D). 


\section{GHET1}

The lncRNA GHET1 is upregulated in ovarian cancer and higher expression correlates with increased tumor size and distant metastasis (Liu and Li, 2019). Conversely, its knockdown induces apoptosis and downregulates glycolysis in A2780 and SKOV3 cells, where GHET1 binds to an E3 ubiquitin ligase, von Hippel-Lindau tumor suppressor (VHL), and prevents VHLmediated degradation of hypoxia-inducible factor $1 \alpha$ (HIF $1 \alpha)$ (Figure 1D). Since the GHET1 function in ovarian cancer cells has been only examined by in vitro assays, in vivo analyses of GHET1 are needed. Although the role of the GHET1/VHL/HIF1 $\alpha$ axis in apoptosis has not yet been elucidated, HIF $1 \alpha$ and cancer metabolism have been shown to play important roles in apoptosis regulation, suggesting the possibility that this axis may also be involved in the phenomenon (Zhou et al., 2006; Matsuura et al., 2016).

\section{NCK1-DT/NCK1-AS1}

In addition to the function as a ceRNA, NCK1-AS1 increases the stability of NCK1: NCK1-AS1 binds to an E3 ubiquitin ligase, casitas B-lineage lymphoma (CBL), and prevents $\mathrm{CBL}$ mediated degradation of NCK1 (Chang et al., 2020; Figure 1D). The functions of NCK-AS1 in ovarian cancer have been suggested based on in vitro experiments, and thus needs to be evaluated using ovarian tumor specimens or in vivo ovarian cancer models.

\section{CONCLUSION}

In this review, we introduced the mechanisms of apoptosisrelated lncRNAs in ovarian cancer cells. Considering that dysregulation of apoptosis is involved in the resistance to ovarian cancer therapies, small molecule inhibitors/siRNAs targeting apoptosis-suppressing lncRNAs, or apoptosispromoting lncRNAs themselves may be applicable to ovarian cancer therapies. For nucleic acid-based therapeutics, it is important to develop the drug delivery systems (DDSs) with high target specificity and less non-specific toxicity in vivo. Particularly, for ovarian cancer, DDSs will be useful to treat metastatic cancer cells in peritoneal cavity (Amreddy et al., 2018; van den Brand et al., 2018). Moreover,

\section{REFERENCES}

Amreddy, N., Babu, A., Muralidharan, R., Panneerselvam, J., Srivastava, A., Ahmed, R., et al. (2018). Recent advances in nanoparticle-based cancer drug and gene delivery. Adv. Cancer Res. 137, 115-170. doi: 10.1016/bs.acr.2017. 11.003

An, J., Lv, W., and Zhang, Y. (2017). LncRNA NEAT1 contributes to paclitaxel resistance of ovarian cancer cells by regulating ZEB1 expression via miR-194. Onco Targets Ther. 10, 5377-5390. doi: 10.2147/OTT.S1 47586

Arun, G., Diermeier, S. D., and Spector, D. L. (2018). Therapeutic targeting of long non-coding RNAs in cancer. Trends Mol. Med. 24, 257-277. doi: 10.1016/ j.molmed.2018.01.001

Beaufort, C. M., Helmijr, J. C., Piskorz, A. M., Hoogstraat, M., Ruigrok-Ritstier, K., Besselink, N., et al. (2014). Ovarian cancer cell line panel (OCCP): clinical importance of in vitro morphological apoptosis-related lncRNAs may be potential diagnostic and prognostic biomarkers. Especially, FEZF1-AS1 and UCA1 are detected in serum and exosomes recovered from serum of ovarian cancer patients, respectively, which suggested their potential as liquid biopsy markers for ovarian cancer.

Apoptosis-related lncRNAs have basically been studied using conventional ovarian cancer cell lines, and the functions of some lncRNAs have been examined by in vitro assays alone. For clinical application, it is required to elucidate the lncRNA functions in vivo. Moreover, previous studies have indicated some discrepancies between ovarian cancer cell lines and the original tumor clinical tissues in terms of genomic and histological features and gene expression profiles (Domcke et al., 2013; Beaufort et al., 2014). Thus, lncRNA studies using ovarian tumor specimens or other ovarian cancer models are strongly demanded. Three-dimensional cultures of patient-derived cancer cells (PDCs) and cancer models established by transplanting tumor specimens into host mice (patient-derived xenograft [PDX] models) retain the properties of original tumors and have attracted attention as promising models for cancer research and drug screening (Ishiguro et al., 2016; Maru and Hippo, 2019; Namekawa et al., 2019; Shiba et al., 2019). Further studies using PDC and PDX models would advance the application of apoptosis-related lncRNAs to ovarian cancer diagnosis, prognosis, and therapies.

\section{AUTHOR CONTRIBUTIONS}

All authors contributed to the conception and provided the data and design. TT contributed to manuscript writing. KI, KH-I, and SI contributed to the conception and final approval of the manuscript.

\section{FUNDING}

This work was supported by grants from the Japan Society for the Promotion of Science (20K21667 to SI, 17H04205 and $20 \mathrm{H} 03734$ to $\mathrm{KH}-\mathrm{I}$, and $20 \mathrm{~K} 21636$ to KI) and Takeda Science Foundation to SI and KI.

subtypes. PLoS One 9:e103988. doi: 10.1371/journal.pone.010 3988

Binju, M., Amaya-Padilla, M. A., Wan, G., Gunosewoyo, H., Suryo Rahmanto, Y., and Yu, Y. (2019). Therapeutic inducers of apoptosis in ovarian cancer. Cancers (Basel) 11:1786. doi: 10.3390/cancers11111786

Bours, V., Bentires-Alj, M., Hellin, A. C., Viatour, P., Robe, P., Delhalle, S., et al. (2000). Nuclear factor-kappa B, cancer, and apoptosis. Biochem. Pharmacol. 60, 1085-1089. doi: 10.1016/s0006-2952(00)00391-9

Braga, E. A., Fridman, M. V., Moscovtsev, A. A., Filippova, E. A., Dmitriev, A. A., and Kushlinskii, N. E. (2020). LncRNAs in ovarian cancer progression, metastasis, and main pathways: ceRNA and alternative mechanisms. Int. J. Mol. Sci. 21:8855. doi: 10.3390/ijms21228855

Bray, F., Ferlay, J., Soerjomataram, I., Siegel, R. L., Torre, L. A., and Jemal, A. (2018). Global cancer statistics 2018: GLOBOCAN estimates of incidence and mortality worldwide for 36 cancers in 185 countries. CA Cancer J. Clin. 68, 394-424. doi: 10.3322/caac.21492 
Cao, L., Bombard, J., Cintron, K., Sheedy, J., Weetall, M. L., and Davis, T. W. (2011). BMI1 as a novel target for drug discovery in cancer. J. Cell. Biochem. 112, 2729-2741. doi: 10.1002/jcb.23234

Chang, H., Li, B., Zhang, X., and Meng, X. (2020). NCK1-AS1 promotes NCK1 expression to facilitate tumorigenesis and chemo-resistance in ovarian cancer. Biochem. Biophys. Res. Commun. 522, 292-299. doi: 10.1016/j.bbrc.2019.11.014

Chen, P., Fang, X., Xia, B., Zhao, Y., Li, Q., and Wu, X. (2018). Long noncoding RNA LINC00152 promotes cell proliferation through competitively binding endogenous miR-125b with MCL-1 by regulating mitochondrial apoptosis pathways in ovarian cancer. Cancer Med. 7, 4530-4541. doi: 10.1002/cam4.1547

Chen, Z. J., Zhang, Z., Xie, B. B., and Zhang, H. Y. (2016). Clinical significance of up-regulated IncRNA NEAT1 in prognosis of ovarian cancer. Eur. Rev. Med. Pharmacol. Sci. 20, 3373-3377.

Christofides, A., Karantanos, T., Bardhan, K., and Boussiotis, V. A. (2016). Epigenetic regulation of cancer biology and anti-tumor immunity by EZH2. Oncotarget 7, 85624-85640. doi: 10.18632/oncotarget.12928

Crosby, M. E., and Almasan, A. (2004). Opposing roles of E2Fs in cell proliferation and death. Cancer Biol. Ther. 3, 1208-1211. doi: 10.4161/cbt.3.12.1494

Di Leva, G., Garofalo, M., and Croce, C. M. (2014). MicroRNAs in cancer. Annu. Rev. Pathol. 9, 287-314. doi: 10.1146/annurev-pathol-012513-104715

Ding, N., Wu, H., Tao, T., and Peng, E. (2017). NEAT1 regulates cell proliferation and apoptosis of ovarian cancer by miR-34a-5p/BCL2. Onco Targets Ther. 10, 4905-4915. doi: 10.2147/OTT.S142446

Domcke, S., Sinha, R., Levine, D. A., Sander, C., and Schultz, N. (2013). Evaluating cell lines as tumour models by comparison of genomic profiles. Nat. Commun. 4:2126. doi: $10.1038 /$ ncomms 3126

El-Deiry, W. S. (2001). Insights into cancer therapeutic design based on p53 and TRAIL receptor signaling. Cell Death Differ. 8, 1066-1075. doi: 10.1038/sj.cdd. 4400943

Gao, J., Liu, M., Zou, Y., Mao, M., Shen, T., Zhang, C., et al. (2015). Long noncoding RNA growth arrest-specific transcript 5 is involved in ovarian cancer cell apoptosis through the mitochondria-mediated apoptosis pathway. Oncol. Rep. 34, 3212-3221. doi: 10.3892/or.2015.4318

Hiller-Sturmhöfel, S., and Bartke, A. (1998). The endocrine system: an overview. Alcohol Health Res. World 22, 153-164.

Hoeller, D., and Dikic, I. (2009). Targeting the ubiquitin system in cancer therapy. Nature 458, 438-444. doi: 10.1038/nature07960

Hu, X., Feng, Y., Zhang, D., Zhao, S. D., Hu, Z., Greshock, J., et al. (2014). A functional genomic approach identifies FAL1 as an oncogenic long noncoding RNA that associates with BMI1 and represses p21 expression in cancer. Cancer Cell 26, 344-357. doi: 10.1016/j.ccr.2014.07.009

Ishiguro, T., Sato, A., Ohata, H., Ikarashi, Y., Takahashi, R. U., Ochiya, T., et al. (2016). Establishment and characterization of an in vitro model of ovarian cancer stem-like cells with an enhanced proliferative capacity. Cancer Res. 76, 150-160. doi: 10.1158/0008-5472.CAN-15-0361

Kamada, S., Takeiwa, T., Ikeda, K., Horie-Inoue, K., and Inoue, S. (2020). Long non-coding RNAs involved in metabolic alterations in breast and prostate cancers. Front. Oncol. 10:593200. doi: 10.3389/fonc.2020.593200

Karin, M. (2006). Nuclear factor- $\kappa \mathrm{B}$ in cancer development and progression. Nature 441, 431-436. doi: 10.1038/nature04870

Kent, L. N., and Leone, G. (2019). The broken cycle: E2F dysfunction in cancer. Nat. Rev. Cancer 19, 326-338. doi: 10.1038/s41568-019-0143-7

Kim, H. S., and Lee, M. S. (2007). STAT1 as a key modulator of cell death. Cell. Signal. 19, 454-465. doi: 10.1016/j.cellsig.2006.09.003

Li, J., Huang, H., Li, Y., Li, L., Hou, W., and You, Z. (2016). Decreased expression of long non-coding RNA GAS5 promotes cell proliferation, migration and invasion, and indicates a poor prognosis in ovarian cancer. Oncol. Rep. 36, 3241-3250. doi: 10.3892/or.2016.5200

Li, Y., Jiao, Y., Hao, J., Xing, H., and Li, C. (2019). Long noncoding RNA TP73-AS1 accelerates the epithelial ovarian cancer via epigenetically repressing p21. Am. J. Transl. Res. 11, 2447-2454.

Li, Z., Niu, H., Qin, Q., Yang, S., Wang, Q., Yu, C., et al. (2019). IncRNA UCA1 mediates resistance to cisplatin by regulating the mir-143/FOSL2-signaling pathway in ovarian cancer. Mol. Ther. Nucleic Acids 17, 92-101. doi: 10.1016/j. omtn.2019.05.007

Li, Z. Y., Wang, X. L., Dang, Y., Zhu, X. Z., Zhang, Y. H., Cai, B. X., et al. (2020). Long non-coding RNA UCA1 promotes the progression of paclitaxel resistance in ovarian cancer by regulating the miR-654-5p/SIK2 axis. Eur. Rev. Med. Pharmacol.Sci. 24, 591-603. doi: 10.26355/eurrev_202001_20035

Liu, D., and Li, H. (2019). Long non-coding RNA GEHT1 promoted the proliferation of ovarian cancer cells via modulating the protein stability of HIF1 $\alpha$. Biosci. Rep. 39:BSR20181650. doi: 10.1042/BSR201 81650

Long, X., Song, K., Hu, H., Tian, Q., Wang, W., Dong, Q., et al. (2019). Long noncoding RNA GAS5 inhibits DDP-resistance and tumor progression of epithelial ovarian cancer via GAS5-E2F4-PARP1-MAPK axis. J. Exp. Clin. Cancer Res. 38:345. doi: 10.1186/s13046-019-1329-2

Luo, P., Liu, X. F., Wang, Y. C., Li, N. D., Liao, S. J., Yu, M. X., et al. (2017). Prognostic value of abnormally expressed lncRNAs in ovarian carcinoma: a systematic review and meta-analysis. Oncotarget 8, 23927-23936. doi: 10.18632/ oncotarget.14760

Mao, B., Zhang, Z., and Wang, G. (2015). BTG2: a rising star of tumor suppressors (review). Int. J. Oncol. 46, 459-464. doi: 10.3892/ijo.2014.2765

Marchese, F. P., Raimondi, I., and Huarte, M. (2017). The multidimensional mechanisms of long noncoding RNA function. Genome Biol. 18:206. doi: 10. 1186/s13059-017-1348-2

Maru, Y., and Hippo, Y. (2019). Current status of patient-derived ovarian cancer models. Cells 8:505. doi: 10.3390/cells8050505

Matsuura, K., Canfield, K., Feng, W., and Kurokawa, M. (2016). Metabolic regulation of apoptosis in cancer. Int. Rev. Cell Mol. Biol. 327, 43-87. doi: 10.1016/bs.ircmb.2016.06.006

Misawa, A., Takayama, K. I., and Inoue, S. (2017). Long non-coding RNAs and prostate cancer. Cancer Sci. 108, 2107-2114. doi: 10.1111/cas.13352

Mitobe, Y., Takayama, K. I., Horie-Inoue, K., and Inoue, S. (2018). Prostate cancerassociated lncRNAs. Cancer Lett. 418, 159-166. doi: 10.1016/j.canlet.2018. 01.012

Momenimovahed, Z., Tiznobaik, A., Taheri, S., and Salehiniya, H. (2019). Ovarian cancer in the world: epidemiology and risk factors. Int. J. Womens Health 11, 287-299. doi: 10.2147/IJWH.S197604

Motta, P. M., Makabe, S., and Nottola, S. A. (1997). The ultrastructure of human reproduction. I. The natural history of the female germ cell: origin, migration and differentiation inside the developing ovary. Hum. Reprod. Update 3, 281295. doi: 10.1093/humupd/3.3.281

Namekawa, T., Ikeda, K., Horie-Inoue, K., and Inoue, S. (2019). Application of prostate cancer models for preclinical study: advantages and limitations of cell lines, patient-derived xenografts, and three-dimensional culture of patientderived cells. Cells 8:74. doi: 10.3390/cells8010074

Pistritto, G., Trisciuoglio, D., Ceci, C., Garufi, A., and D’Orazi, G. (2016). Apoptosis as anticancer mechanism: function and dysfunction of its modulators and targeted therapeutic strategies. Aging (Albany NY) 8, 603-619. doi: 10.18632/ aging.100934

Qiu, J. J., Wang, Y., Ding, J. X., Jin, H. Y., Yang, G., and Hua, K. Q. (2015). The long non-coding RNA HOTAIR promotes the proliferation of serous ovarian cancer cells through the regulation of cell cycle arrest and apoptosis. Exp. Cell Res. 333, 238-248. doi: 10.1016/j.yexcr.2015.03.005

Qu, C., Dai, C., Guo, Y., Qin, R., and Liu, J. (2020). Long non-coding RNA PVT1mediated miR-543/SERPINI1 axis plays a key role in the regulatory mechanism of ovarian cancer. Biosci. Rep. 40:BSR20200800. doi: 10.1042/BSR20200800

Rojas, V., Hirshfield, K. M., Ganesan, S., and Rodriguez-Rodriguez, L. (2016). Molecular characterization of epithelial ovarian cancer: implications for diagnosis and treatment. Int. J. Mol. Sci. 17:2113. doi: 10.3390/ijms17122113

Ruan, Z., and Zhao, D. (2019). Long intergenic noncoding RNA LINC00284 knockdown reduces angiogenesis in ovarian cancer cells. FASEB J. 33, 1204712059. doi: 10.1096/fj.201900101RR

Rupaimoole, R., Lee, J., Haemmerle, M., Ling, H., Previs, R. A., Pradeep, S., et al. (2015). Long noncoding RNA ceruloplasmin promotes cancer growth by altering glycolysis. Cell Rep. 13, 2395-2402. doi: 10.1016/j.celrep.2015. 11.047

Salamini-Montemurri, M., Lamas-Maceiras, M., Barreiro-Alonso, A., VizosoVázquez, Á, Rodríguez-Belmonte, E., Quindós-Varela, M., et al. (2020). The challenges and opportunities of lncRNAs in ovarian cancer research and clinical use. Cancers (Basel) 12:1020. doi: 10.3390/cancers12041020

Shiba, S., Ikeda, K., Suzuki, T., Shintani, D., Okamoto, K., Horie-Inoue, K., et al. (2019). Hormonal regulation of patient-derived endometrial cancer stem-like 
cells generated by three-dimensional culture. Endocrinology 160, 1895-1906. doi: 10.1210/en.2019-00362

Si, W., Shen, J., Zheng, H., and Fan, W. (2019). The role and mechanisms of action of microRNAs in cancer drug resistance. Clin. Epigenetics 11:25. doi: 10.1186/s13148-018-0587-8

Siegel, R. L., Miller, K. D., and Jemal, A. (2018). Cancer statistics, 2018. CA Cancer J. Clin. 68, 7-30. doi: 10.3322/caac. 21442

Sun, Z., Gao, S., Xuan, L., and Liu, X. (2020). Long non-coding RNA FEZF1-AS1 induced progression of ovarian cancer via regulating miR-130a-5p/SOX4 axis. J. Cell. Mol. Med. 24, 4275-4285. doi: 10.1111/jcmm.15088

Takayama, K., and Inoue, S. (2016). The emerging role of noncoding RNA in prostate cancer progression and its implication on diagnosis and treatment. Brief. Funct. Genomics 15, 257-265. doi: 10.1093/bfgp/elv057

Takeiwa, T., Ikeda, K., Mitobe, Y., Horie-Inoue, K., and Inoue, S. (2020). Long noncoding rnas involved in the endocrine therapy resistance of breast cancer. Cancers (Basel) 12:1424. doi: 10.3390/cancers12061424

Tao, P., Yang, B., Zhang, H., Sun, L., Wang, Y., and Zheng, W. (2020). The overexpression of lncRNA MEG3 inhibits cell viability and invasion and promotes apoptosis in ovarian cancer by sponging miR-205-5p. Int. J. Clin. Exp. Pathol. 13, 869-879.

Torre, L. A., Trabert, B., DeSantis, C. E., Miller, K. D., Samimi, G., Runowicz, C. D., et al. (2018). Ovarian cancer statistics, 2018. CA Cancer J. Clin. 68, 284-296. doi: $10.3322 /$ caac. 21456

Trinidad, C. V., Tetlow, A. L., Bantis, L. E., and Godwin, A. K. (2020). Reducing ovarian cancer mortality through early detection: approaches using circulating biomarkers. Cancer Prev. Res. (Phila.) 13, 241-252. doi: 10.1158/1940-6207. CAPR-19-0184

van den Brand, D., Mertens, V., Massuger, L. F. A. G., and Brock, R. (2018). siRNA in ovarian cancer - delivery strategies and targets for therapy. J. Control. Release 283, 45-58. doi: 10.1016/j.jconrel.2018.05.012

Vaskivuo, T. E., and Tapanainen, J. S. (2003). Apoptosis in the human ovary. Reprod. Biomed. Online 6, 24-35. doi: 10.1016/s1472-6483(10)62052-4

Verhoeven, Y., Tilborghs, S., Jacobs, J., De Waele, J., Quatannens, D., Deben, C., et al. (2020). The potential and controversy of targeting STAT family members in cancer. Semin. Cancer Biol. 60, 41-56. doi: 10.1016/j.semcancer.2019.10.002

Virant-Klun, I. (2015). Postnatal oogenesis in humans: a review of recent findings. Stem Cells Cloning 8, 49-60. doi: 10.2147/SCCAA.S32650

Wang, H., Su, H., and Tan, Y. (2020). UNC5B-AS1 promoted ovarian cancer progression by regulating the $\mathrm{H} 3 \mathrm{~K} 27 \mathrm{me}$ on NDRG2 via EZH2. Cell Biol. Int. 44, 1028-1036. doi: 10.1002/cbin. 11300

Wang, J., Ye, C., Liu, J., and Hu, Y. (2018). UCA1 confers paclitaxel resistance to ovarian cancer through miR-129/ABCB1 axis. Biochem. Biophys. Res. Commun. 501, 1034-1040. doi: 10.1016/j.bbrc.2018.05.104

Wang, W., Qin, J. J., Voruganti, S., Nag, S., Zhou, J., and Zhang, R. (2015). Polycomb group ( $\mathrm{PcG}$ ) proteins and human cancers: multifaceted functions and therapeutic implications. Med. Res. Rev. 35, 1220-1267. doi: 10.1002/med. 21358

Wang, Y., Wang, H., Song, T., Zou, Y., Jiang, J., Fang, L., et al. (2015). HOTAIR is a potential target for the treatment of cisplatin-resistant ovarian cancer. Mol. Med. Rep. 12, 2211-2216. doi: 10.3892/mmr.2015.3562

Wong, R. S. (2011). Apoptosis in cancer: from pathogenesis to treatment. J. Exp. Clin. Cancer Res. 30:87. doi: 10.1186/1756-9966-30-87

Woo, S. M., and Kwon, T. K. (2019). E3 ubiquitin ligases and deubiquitinases as modulators of TRAIL-mediated extrinsic apoptotic signaling pathway. BMB Rep. 52, 119-126. doi: 10.5483/BMBRep.2019.52.2.011

Wu, D. D., Chen, X., Sun, K. X., Wang, L. L., Chen, S., and Zhao, Y. (2017). Role of the IncRNA ABHD11-AS1 in the tumorigenesis and progression of epithelial ovarian cancer through targeted regulation of RhoC. Mol. Cancer 16:138. doi: 10.1186/s12943-017-0709-5

Xu, H., Sun, X., Huang, Y., Si, Q., and Li, M. (2020). Long non-coding RNA NEAT1 modifies cell proliferation, colony formation, apoptosis, migration and invasion via the miR-4500/BZW1 axis in ovarian cancer. Mol. Med. Rep. 22, 3347-3357. doi: 10.3892/mmr.2020.11408

Yadav, P. K., Tiwari, M., Gupta, A., Sharma, A., Prasad, S., Pandey, A. N., et al. (2018). Germ cell depletion from mammalian ovary: possible involvement of apoptosis and autophagy. J. Biomed. Sci. 25:36. doi: 10.1186/s12929-0180438-0

Yang, L., Chen, J., Huang, X., Zhang, E., He, J., and Cai, Z. (2018). Novel insights into E3 ubiquitin ligase in cancer chemoresistance. Am. J. Med. Sci. 355, 368-376. doi: 10.1016/j.amjms.2017.12.012

Yang, Y., Kitagaki, J., Wang, H., Hou, D. X., and Perantoni, A. O. (2009). Targeting the ubiquitin-proteasome system for cancer therapy. Cancer Sci. 100, 24-28. doi: 10.1111/j.1349-7006.2008.01013.x

Ye, F., Chen, C., Qin, J., Liu, J., and Zheng, C. (2015). Genetic profiling reveals an alarming rate of cross-contamination among human cell lines used in China. FASEB J. 29, 4268-4272. doi: 10.1096/fj.14-266718

Yu, Y., Zhang, X., Tian, H., Zhang, Z., and Tian, Y. (2018). Knockdown of long non-coding RNA HOTAIR increases cisplatin sensitivity in ovarian cancer by inhibiting cisplatin-induced autophagy. J. BUON 23, 1396-1401.

Zeng, X. Y., Jiang, X. Y., Yong, J. H., Xie, H., Yuan, J., Zeng, D., et al. (2019). IncRNA ABHD11-AS1, regulated by the EGFR pathway, contributes to the ovarian cancer tumorigenesis by epigenetically suppressing TIMP2. Cancer Med. 8, 7074-7085. doi: 10.1002/cam4.2586

Zhang, H. G., Wang, J., Yang, X., Hsu, H. C., and Mountz, J. D. (2004). Regulation of apoptosis proteins in cancer cells by ubiquitin. Oncogene 23, 2009-2015. doi: 10.1038/sj.onc. 1207373

Zhang, Y., Ai, H., Fan, X., Chen, S., Wang, Y., and Liu, L. (2020). Knockdown of long non-coding RNA HOTAIR reverses cisplatin resistance of ovarian cancer cells through inhibiting miR-138-5p-regulated EZH2 and SIRT1. Biol. Res. 53:18. doi: 10.1186/s40659-020-00286-3

Zhang, Z., Cheng, J., Wu, Y., Qiu, J., Sun, Y., and Tong, X. (2016). LncRNA HOTAIR controls the expression of Rab22a by sponging miR-373 in ovarian cancer. Mol. Med. Rep. 14, 2465-2472. doi: 10.3892/mmr.2016.5572

Zhao, H., Yu, H., Zheng, J., Ning, N., Tang, F., Yang, Y., et al. (2018). Lowlyexpressed lncRNA GAS5 facilitates progression of ovarian cancer through targeting miR-196-5p and thereby regulating HOXA5. Gynecol. Oncol. 151, 345-355. doi: 10.1016/j.ygyno.2018.08.032

Zhou, J., Schmid, T., Schnitzer, S., and Brüne, B. (2006). Tumor hypoxia and cancer progression. Cancer Lett. 237, 10-21. doi: 10.1016/j.canlet.2005.05.028

Zhu, M., Yang, L., and Wang, X. (2020). NEAT1 knockdown suppresses the cisplatin resistance in ovarian cancer by regulating miR-770 5p/PARP1 axis. Cancer Manag. Res. 12, 7277-7289. doi: 10.2147/CMAR.S2 57311

Conflict of Interest: The authors declare that the research was conducted in the absence of any commercial or financial relationships that could be construed as a potential conflict of interest.

Copyright (C) 2021 Takeiwa, Ikeda, Horie-Inoue and Inoue. This is an open-access article distributed under the terms of the Creative Commons Attribution License (CC BY). The use, distribution or reproduction in other forums is permitted, provided the original author(s) and the copyright owner(s) are credited and that the original publication in this journal is cited, in accordance with accepted academic practice. No use, distribution or reproduction is permitted which does not comply with these terms. 